\title{
Effect of Magnetic Field on Flow, Temperature and Inclusion Removal in Shallow Tundishes
}

\section{O.J. ILEGBUSI and J. SZEKELY}

Department of Materials Science and Engineering, Massachusetts Institute of Technology, Cambridge, MA 02139, U.S.A.

(Received on March 9, 1989; accepted in the final form on July 21, 1989)

\begin{abstract}
A mathematical representation has been developed to describe the three-dimensional velocity and temperature fields in shallow tundishes to be used in novel continuous casting systems. Particular attention has been paid to the effect of externally imposed magnetic fields in modifying the flow. It was found that the imposition of vertical or horizontal fields may play an important role in minimizing flow non-uniformities and hence variations in the temperature of the stream entering the mold of the caster. In the absence of (magnetic) flow control arrangements, serious flow and temperature maldistribution may occur, especially in the production of wide strip or thin slabs. The effect of magnetic fields on inclusion removal has also been investigated, but the inclusion removal efficiency of shallow tundishes is thought to be low in any case.
\end{abstract}

KEY WORDS: tundish; fluid flow; heat transfer; inclusion; magnetohydrodynamics.

\section{Introduction}

In recent years, there has been a growing interest in near-net shape casting processes, which allow the transformation of molten steel into thin slabs or sheet. Indeed, several symposia have been devoted to this topic. Most of the work reported to date such as those in references ${ }^{1-8}$ ) has been concerned with the description of experimental equipment, the heat transfer analysis and the microstructural evaluation of the products. It is now being realized that just as with conventional continuous casting systems, the mode of metal delivery through tundishes may play a key role in determining the overall performance of these casting units. Indeed, some recently published papers by the authors ${ }^{4,5}$ have indicated that problems in flow maldistribution may be even more critical in tundishes supplying near-net shape casting systems, particularly those concerned with production of wide sheet and plate.

The reasons for the critical role of tundish flow in these systems are quite simple and may be summarized as follows:

(1) Near-net shape casting systems tend to be much less "forgiving" regarding flow maldistribution and uneven temperatures in the stream entering the mold, because the solidification time is very much shorter and the depth of the molten pool or molten regions tends to be much smaller.

(2) The tundishes themselves will by necessity tend to be much shallower and of much smaller capacity, so their role as a buffer will be much less effective. A logical consequence of this state of affairs is that the conditions of introduction into the mold and inflow into the tundish itself may play a much more important role here.
In previous publications, ${ }^{4-6)}$ the authors have presented a general formulation of the fluid flow and heat transfer problem in the operation of shallow tundishes and have shown that quite severe uneven flow may take place unless the tundishes are carefully designed. This earlier work has also indicated that the imposition of a (horizontal) magnetic field, perpendicular to the principal flow direction, may be quite effective in minimizing melt flow maldistribution.

In the present paper, we shall seek to extend the scope of these ideas by examining:

1) the behavior of tundishes to be employed in the casting of wide (thin) slabs, a problem of major practical importance.

2) the effect of magnetic field orientation and the potential role of a variable magnetic field.

3 ) the explicit effect of melt flow maldistribution on the horizontal variations in the melt temperature, particularly in the exit region of the tundish.

4) the effect of these parameters on the flotation behavior of inclusion particles.

\section{Mathematical Formulation}

Fig. 1 shows a schematic of a typical shallow tundish into which molten metal is fed vertically at one end and is being discharged through a slot at the other. This arrangement, even though idealized, would approximate the tundishes employed in belt casters and single-roll casters.

In order to determine the fluid flow, heat transfer and inclusion behavior, we need to solve three-dimensional transport equations, coupled with equations to represent the electromagnetic force field and associated Joule heating, and the appropriate bound- 


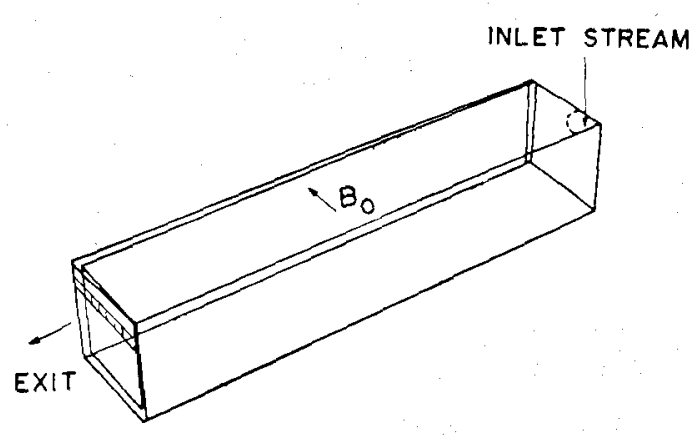

Fig. 1. A schematic sketch of a shallow tundish with transverse magnetic field $B_{o}$.

ary conditions.

The system of equations are expressed as follows: Equation of Continuity

$$
\frac{\partial \rho u}{\partial x}+\frac{\partial \rho v}{\partial y}+\frac{\partial \rho w}{\partial z}=0
$$

Equation of Motion in $x$-direction

$$
\begin{aligned}
\frac{\partial \rho u^{2}}{\partial x} & +\frac{\partial \rho u v}{\partial y}+\frac{\partial \rho u w}{\partial z} \\
= & -\frac{\partial p}{\partial x}+\frac{\partial}{\partial x}\left(\mu_{\mathrm{eff}} \frac{\partial u}{\partial x}\right)+\frac{\partial}{\partial y}\left(\mu_{\mathrm{eff}} \frac{\partial u}{\partial y}\right) \\
& +\frac{\partial}{\partial z}\left(\mu_{\mathrm{eff}} \frac{\partial u}{\partial z}\right)+F_{\text {ex }}
\end{aligned}
$$

Equation of Motion in $y$-direction

$$
\begin{aligned}
\frac{\partial \rho u v}{\partial x} & +\frac{\partial \rho v^{2}}{\partial y}+\frac{\partial \rho v w}{\partial z} \\
= & -\frac{\partial p}{\partial y}+\frac{\partial}{\partial x}\left(\mu_{\text {eff }} \frac{\partial v}{\partial x}\right)+\frac{\partial}{\partial y}\left(\mu_{\text {eff }} \frac{\partial v}{\partial y}\right) \\
& +\frac{\partial}{\partial z}\left(\mu_{\text {eff }} \frac{\partial v}{\partial z}\right)+F_{e y}
\end{aligned}
$$

Equation of Motion in $z$-direction

$$
\begin{aligned}
\frac{\partial \rho u w}{\partial x}+\frac{\partial \rho v w}{\partial y}+\frac{\partial \rho w^{2}}{\partial z} \\
=-\frac{\partial p}{\partial z}+\frac{\partial}{\partial x}\left(\mu_{\mathrm{eff}} \frac{\partial w}{\partial x}\right)+\frac{\partial}{\partial y}\left(\mu_{\mathrm{eff}} \frac{\partial u}{\partial y}\right) \\
\quad+\frac{\partial}{\partial z}\left(\mu_{\mathrm{eff}}^{\frac{\partial}{\partial z}}\right)+F_{e z}+\rho g
\end{aligned}
$$

Thermal Energy Balance

$$
\begin{aligned}
& \frac{\partial \rho u T}{\partial x}+\frac{\partial \rho v T}{\partial y}+\frac{\partial \rho w T}{\partial z} \\
& =\frac{\mu_{\text {eff }}}{\sigma} \frac{\partial^{2} \mathcal{T}}{\partial x^{2}}+\frac{\mu_{\text {eff }}}{\sigma} \frac{\partial^{2} T}{\partial y^{2}}+\frac{\mu_{\text {eff }}}{\sigma} \frac{\partial^{2} T}{\partial z^{2}}+\frac{|J|^{2}}{\kappa c_{p}}
\end{aligned}
$$

\section{Transport of Inclusions}

In establishing a material balance on the inclusions, we have made allowance for their generation and destruction by coalescence. By considering a population balance for inclusion particles of the same group characterized principally by their size, we have:

$$
\begin{aligned}
& \frac{\partial \rho C_{i}}{\partial t}+\frac{\partial \rho u C_{i}}{\partial x}+\frac{\partial \rho v C_{i}}{\partial y}+\frac{\partial \rho\left(w+w_{p i}\right) C_{i}}{\partial z} \\
& \quad=\frac{\mu_{\mathrm{efr}}}{\sigma_{c i}} \frac{\partial^{2} C_{i}}{\partial x^{2}}+\frac{\mu_{\mathrm{eff}}}{\sigma_{c i}} \frac{\partial^{2} C_{i}}{\partial y^{2}}+\frac{\mu_{\mathrm{eff}}}{\sigma_{c i}} \frac{\partial^{2} C_{i}}{\partial z^{2}}+S_{i}
\end{aligned}
$$

where, $C_{i}$ : the number density i.e. number of particles of type $i$ per unit volume

$S_{i}$ : the sum of the source and sink terms in the population balance for particles of type $i$

$w_{p i}$ : the terminal rising velocity of particles of type $i$ which depends on the density and diameter. The addition of $w_{p i}$ to the vertical component of velocity in Eq. (6) characterizes the direct effect of buoyancy on the particles

$\sigma_{c i}$ : Schmidt number of particles of type $i$. As detailed in Soo, ${ }^{71} \sigma_{{ }^{c} i}$ depends on the Langrangian and Eulerian microscales, density ratio and particle size. However, Shirabe ${ }^{8 !}$ has shown that $\sigma_{c i}$ is approximately unity for particles suspended in steel. This value has been adopted in the present work. This choice is not expected to alter markedly the overall character of the flow and the main conclusions derived from it.

In representing $S_{i}$, we use the approach of Shirabe ${ }^{8)}$ and Shirabe and Szekely9) in which the specific expression that results even in the absence of nucleation, is a complex integro-differential equation. Details of this model will not be reproduced here. However, it should be mentioned that $S_{i}$ depends on the rate of coalescence between the particles. If we assume that two spherical particles coalesce to form another spherical particle, the coalescence frequency can be expressed in terms of the turbulence quantities by the following relationship developed by Saffman and Turner ${ }^{10)}$ :

$$
f_{i j}=1.6 R_{i j}^{3} C_{i} C_{j}\left[\frac{\varepsilon}{\nu}\right]^{1 / 2}
$$

where, $f_{i j}$ : coalescence frequency between particles of types $i$ and $j$ per unit time and per unit volume

$$
\begin{aligned}
R_{i j}: & r_{i}+r_{j} \\
\varepsilon: & \text { rate of dissipation of turbulence energy } \\
\nu: & \text { kinematic viscosity. }
\end{aligned}
$$

The characteristic " rising" velocity $w_{p i}$ is calculated from the following relationship obtained by balancing the force of gravity and the frictional drag on the particle (i.e., Stoke's law):

$$
w_{p i}=\frac{2 r_{i}^{2} g\left(\rho_{p i}-\rho\right)}{9 \mu}
$$

where, $r_{i}$ : radius of particles of type $i$

$\rho_{p i}:$ density of particles of type $i$

$\rho$ : melt density.

In Eqs. (2) to (5), the effective viscosity $\mu_{\text {eff }}$ is defined as: 


$$
\mu_{\mathrm{eff}}=\mu+\mu_{t}
$$

where, $\mu, \mu_{t}$ : the molecular and the eddy viscosities, respectively.

The latter is expressed in terms of $k$, the turbulent energy, and $\varepsilon$, its rate of dissipation thus:

$$
\mu_{t}=c_{\mu} \rho k^{2} / \varepsilon
$$

where, $c_{\mu}$ : an empirical constant $(=0.09)$.

The turbulence quantities $k$ and $\varepsilon$ are obtained by solving transport equations as discussed by Launder and Spalding. ${ }^{11)}$

The electromagnetic force $F_{e} \equiv\left(F_{e x}, F_{e y}, F_{e z}\right)$ is calculated from the Maxwell relation:

$$
F_{e}=J \times B
$$

where, $B$ : the magnetic field

$J:$ the induced current density.

The exact expressions for the components $F_{e x}, F_{e y}$ and $F_{e z}$ of $F_{c}$ will of course depend on both the magnitude and orientation of $B$. As an example, the expressions for the case of a constant transverse (horizontal) field $B_{o}$ oriented parallel to the $y$-axis are:

$$
\begin{aligned}
& F_{e x}=-\kappa\left(E_{z} B_{o}+u B_{o}^{2}\right) \\
& F_{e y}=0 \ldots \ldots \ldots \ldots \ldots \ldots \ldots \ldots \ldots \ldots \\
& F_{e z}=\kappa\left(E_{x} B_{o}-w B_{o}^{2}\right)
\end{aligned}
$$

where, $E_{x}, E_{z}$ : the $x$ and $z$ components of the electric field $E$, respectively

$\kappa$ : the electrical conductivity.

The latter are obtained by defining an electric potential $\phi$ such that:

$$
E=-\nabla \phi
$$

From Maxwell's relations, we can show that:

$$
\nabla^{2} \phi=B_{o}(\nabla \cdot U)
$$

Eqs. (15) and (16) are solved for $E$ from which $F_{e}$ can be obtained using Eqs. (12) to (14). It should be noted that Eq. (15) implies that the solution of the electromagnetic force field and flow field are directly coupled.

For the particular magnetic field orientation considered, the current density components used for calculating the Joule heating are obtained from Maxwell's relations and are expressed as:

$$
\begin{aligned}
& J_{x}=\kappa\left[-\frac{\partial \phi}{\partial x}-w B_{o}\right] \\
& J_{y}=-\kappa \frac{\partial \phi}{\partial y} \cdots \cdots \cdots . . . \\
& J_{z}=\kappa\left[-\frac{\partial \phi}{\partial z}+u B_{o}\right]
\end{aligned}
$$

The boundary conditions employed in the computations can be summarized as follows:

(1) A uniform velocity profile equal to the value given in Table 1 is specified at the inlet nozzle. This velocity is oriented downwards (i.e., negative $z$-direction) as shown in Fig. 1.

(2) A no-slip condition is imposed at the solid surfaces through a logarithmic wall function similar to that described by Launder and Spalding. ${ }^{12)}$

(3) The free surface and symmetry planes are assumed to be stress free.

(4) Thermal radiation is allowed through the top surface which is assumed to be covered by $15 \mathrm{~mm}$ thick slag in all cases.

(5) All boundaries are assumed to be electrically insulated.

(6) The inclusion particles are assumed to be ideally absorbed at the free surface. This condition can be expressed for particles of type $i$ by the relation:

$$
q_{i}=w_{p i} C_{i s}
$$

where, $C_{i s}$ : the number density of such particles just below the surface

$q_{i}$ : the flux density of particles of type $i$ at the surface, i.e. the number of such particles leaving the surface per unit area per unit time.

It should be remarked that the above model has been employed by Tacke and Ludwig $^{13}$ albeit in a more restricted sence. These authors considered inclusions of the same size and there was no allowance for inclusion coalescence and of course, the influence of magnetic field on the melt motion. However, their work provided a useful basis for the calculations reported here. Essentially, we assume a certain initial distribution of inclusion particles of known sizes and then compute their behavior both in the absence and presence of magnetic field.

The principal input parameters of the calculations are given in Table 1. In Table 2 we summarized the inclusion sizes considered and their initial number densities.

\section{Computational Technique}

The transport equations are solved using a fullyimplicit, finite-domain numerical technique embodied

Table 1. Principal input parameters.

\begin{tabular}{lll}
\multicolumn{1}{c}{ Parameter } & Cases 1-2 & Cases 3-6 \\
\hline Tundish dimensions & $1 \mathrm{~m} \times 0.5 \mathrm{~m} \times$ & $1 \mathrm{~m} \times 1 \mathrm{~m} \times$ \\
& $0.25 \mathrm{~m}$ & $0.125 \mathrm{~m}$ \\
Inlet nozzle diameter & $40 \mathrm{~mm}$ & $60 \mathrm{~mm}$ \\
Outlet slot height & $25 \mathrm{~mm}$ & $12.5 \mathrm{~mm}$ \\
Inlet flow velocity & $0.25 \mathrm{~m} / \mathrm{s}$ & $0.25 \mathrm{~m} / \mathrm{s}$ \\
Inlet temperature & $1873 \mathrm{~K}$ & $1873 \mathrm{~K}$ \\
Inclusion density & $2700 \mathrm{~kg} / \mathrm{m}^{3}$ & $2700 \mathrm{~kg} / \mathrm{m}^{3}$ \\
\hline
\end{tabular}

Table 2. Inclusion sizes and initial number densities.

\begin{tabular}{ccl}
\hline Particle type & $\begin{array}{c}\text { Diameter } \\
\left(\times 10^{6} \mathrm{~m}\right)\end{array}$ & $\begin{array}{c}\text { Initial density } \\
\left(\mathrm{No} / \mathrm{m}^{3}\right)\end{array}$ \\
\hline $\mathrm{C}_{1}$ & 100 & $10^{12}$ \\
$\mathrm{C}_{2}$ & 80 & $2.575 \times 10^{13}$ \\
$\mathrm{C}_{3}$ & 60 & $5.05 \times 10^{13}$ \\
$\mathrm{C}_{4}$ & 40 & $7.525 \times 10^{13}$ \\
$\mathrm{C}_{5}$ & 20 & $10^{14}$ \\
\hline
\end{tabular}


in the PHOENIGS computer code of Spalding. ${ }^{14)}$ This technique derives from the SIMPLE algorithm of Patankar and Spalding. ${ }^{15}$ ) The electromagnetic phenomena are however calculated by an internallygenerated code independent of PHOENICS. The two codes are coupled at every iteration of the numerical scheme through the velocity field.

A $25 \times 15 \times 15$ grid was found to be adequate for one half of the tundish. The numerical accuracy of this grid was ascertained by the fact that relatively insignificant changes occur in the values of the calculated variables by a finer grid structure.

Each run required about 100 sweeps of the computational domain and is terminated when the residual continuity error was less than $1 \%$ of the input mass. Each of these runs took about $4 \mathrm{~h}$ on a MicroVax II. In order to reduce computation cost, the transient inclusion transport equation is solved after the velocity field has fully converged.

\section{Computed Results}

In the following, we shall present a selection of the computed results. Six specific cases have been considered, as summarized in Table 3. The first two cases refer to the behavior of a small tundish, $1 \mathrm{~m}$ long, $0.5 \mathrm{~m}$ wide and $0.25 \mathrm{~m}$ deep, in the absence and in the presence of vertically-oriented constant magnetic field.

The second group of four cases refers to a larger tundish, $1 \mathrm{~m}$ long, $1 \mathrm{~m}$ wide, and $0.125 \mathrm{~m}$ deep. Here, we considered the absence of a magnetic field, a uniform vertical field and variable vertical and horizontal fields.

It should be remarked here that in the plots that will be presented, only the behavior of half the tundish is considered because of symmetry.

Figs. 2 and 3 show computed velocity fields for case 1, i.e. small tundish in the absence of a magnetic field, in two horizontal planes. The marked recirculation is readily apparent in both these plots. As expected, the recirculation is more pronounced in the plane close to the bottom, because the stream has to "bend over" and be redistributed in this region.

Figs. 4 and 5 show the corresponding temperature profiles, which indicate the existence of significant horizontal temperature variations, even for the relatively narrow tundish. Such temperature variations could be particularly detrimental in single roll casting or melt extraction systems. One should note that while the absolute values of the temperature loss may

Table 3. The cases considered.

\begin{tabular}{|c|c|}
\hline $\begin{array}{l}\text { Case } \\
\text { No. }\end{array}$ & Description \\
\hline 1 & $\begin{array}{l}\text { Narrow tundish }(1 \mathrm{~m} \times 0.5 \mathrm{~m} \times 0.25 \mathrm{~m}) \text { without magnetic } \\
\text { field. }\end{array}$ \\
\hline 2 & $\begin{array}{l}\text { Narrow tundish }(1 \mathrm{~m} \times 0.5 \mathrm{~m} \times 0.25 \mathrm{~m}) \text { with constant } \\
\text { magnetic field of } 1 \mathrm{kGauss} \text { oriented in the vertical }(z) \\
\text { direction. }\end{array}$ \\
\hline 3 & $\begin{array}{l}\text { Wide tundish ( } 1 \mathrm{~m} \times 1 \mathrm{~m} \times 0.125 \mathrm{~m} \text { ) without magnetic } \\
\text { field. }\end{array}$ \\
\hline 4 & $\begin{array}{l}\text { Wide tundish }(1 \mathrm{~m} \times 1 \mathrm{~m} \times 0.125 \mathrm{~m}) \text { with constant mag- } \\
\text { netic field of } 1 \mathrm{kGauss} \text { oriented in the vertical }(z) \\
\text { direction. }\end{array}$ \\
\hline 5 & $\begin{array}{l}\text { Wide tundish }(1 \mathrm{~m} \times 1 \mathrm{~m} \times 0.125 \mathrm{~m}) \text { with vertical }(z \text {-di. } \\
\text { rected) magnetic field varying from } 1 \mathrm{kGauss} \text { at inlet } \\
\text { to } 0 \text { at exit. }\end{array}$ \\
\hline 6 & $\begin{array}{l}\text { Wide tundish }(1 \mathrm{~m} \times 1 \mathrm{~m} \times 0.125 \mathrm{~m}) \text { with horizontal }(y \text { - } \\
\text { directed) magnetic field varying from } 1 \mathrm{kGauss} \text { at inlet } \\
\text { to } 0 \text { at exit. }\end{array}$ \\
\hline
\end{tabular}

Fig. 2.

Velocity vectors at exit plane $(z=0.75$ $H$ ) for case 1.

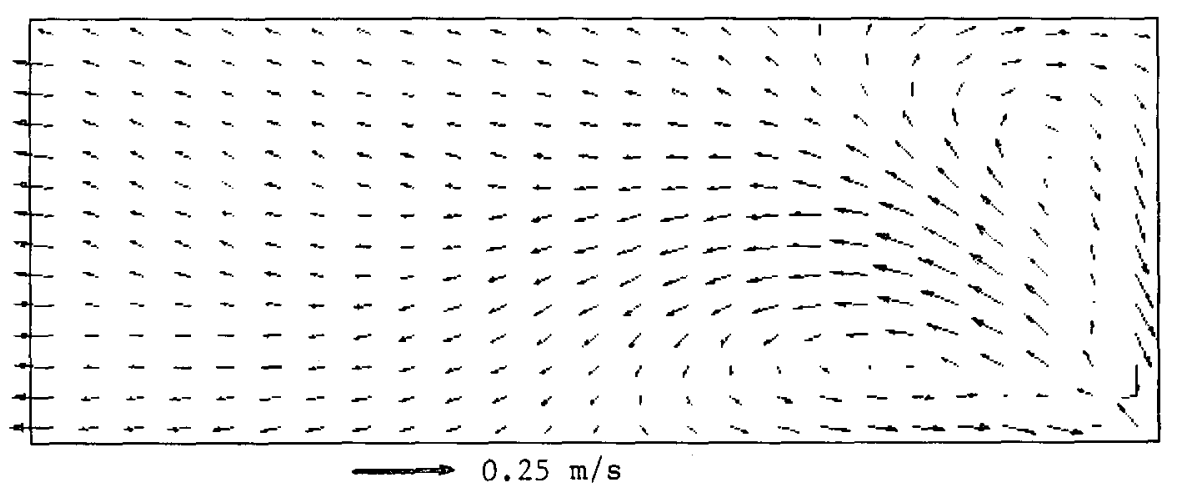

Fig. 3.

Velocity vectors near bottom plane $(z=$ $0.1 H$ ) for case 1 . 
Fig. 4.

Map of temperature at exit plane $(z=$

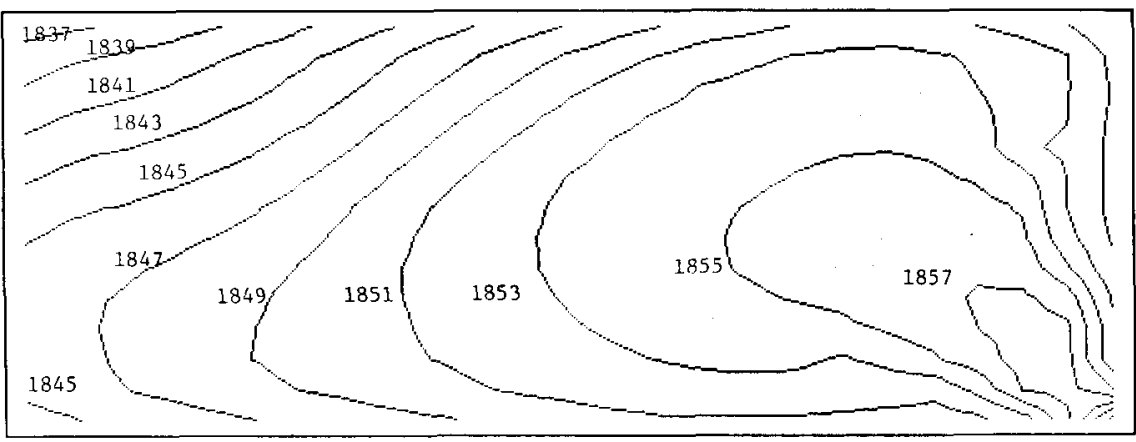

$0.75 H)$ for case 1 .

1872

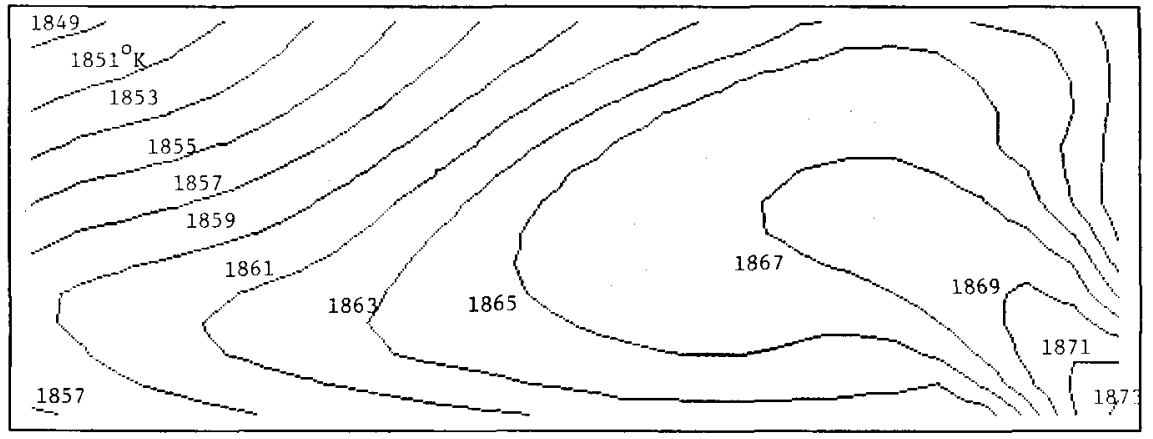

Fig. 5.

Map of temperature near bottom plane $(z=0.1 H)$ for case 1 . depend on the boundary conditions imposed at the top surface (thickness of slag cover, radiation shiclds, auxiliary heating, temperature of the surroundings), the relative magnitude of the horizontal temperature variations should be generally indicative of the behavior of most systems of interest.

Figs. 6 to 9 show the corresponding plots, but now with a vertical magnetic field of $1 \mathrm{kGauss}$ strength imposed on the system. These calculations should be of practical interest, because the imposition of such a vertical field should be much easier to construct than the use of horizontal fields, discussed in our earlier publications.

The computed results show the essentially expected results in that the magnetic field will tend to "straighten out" the flow, eliminate recirculation and reduce the temperature differences across the tundish.

Calculations have also been carried out to examine the behavior of a larger tundish, $1 \mathrm{~m}$ long, $1 \mathrm{~m}$ wide and $0.125 \mathrm{~m}$ deep (cases 3-6). These problems are of considerable practical interest, because ultimately, the much narrower laboratory-scale casting systems will have to be scaled up to produce strip or thin slabs that are say 1-2 $\mathrm{m}$ wide. If possible, the use of very long tundishes should be avoided, because of the very high losses inherently associated with the large surface areas that such systems represent.

The velocity fields and temperature maps calculated for these cases are not reproduced here, for the case of brevity. We note however, that with the shallower depths and the one to one length to width ratios, recirculation and flow maldistribution were rather more serious than seen in the previous seven figures.

In the following Figs. 10 and 11, we shall illustrate the key effect of flow maldistribution by plotting the horizontal variation of the temperature at the exit and the vertical variation of the temperature at the midplane $(x=0.5 L)$ of the tundish. Both these plots are quite instructive, because they clearly indicate the marked effect of the magnetic field on the temperature uniformities in the system.

Fig. 10 shows that very serious temperature variations will exist in the absence of a magnetic field and that case 6 with a variable horizontal field appears to provide the most uniform temperature. It should be stressed that the virtually heat-loss-free operation found for case 6 is due to the marked suppression of vertical flow, together with the specific thermal boundary conditions imposed on the system. Nevertheless, in the cases considered the imposition of a vertical or horizontal field will tend to improve the uniformity of the temperature at the exit. This is an important finding, because it may allow one to readily optimize the tundish design so that the dual objectives of minimal heat loss and temperature uniformity at the exit are accomplished.

Fig. Il shows the vertical temperature variations in the midplane of the tundish. Here again, case 6 , that is, a variable transverse field provides the highest degree of uniformity while the absence of the field (case 3) and a variable vertical field (case 5) appear to provide the greatest variations in temperature. This behavior is consistent with physical reasoning because the vertical field has no significant effect in reducing the vertical velocity components (except through continuity), which are responsible for transporting high-temperature fluid from the bottom to the surface.

In case 4 , substantial heat has been lost prior to the midplane location considered for the same reason. At this location, the temperature remains approximately constant vertically partly due to its smaller 


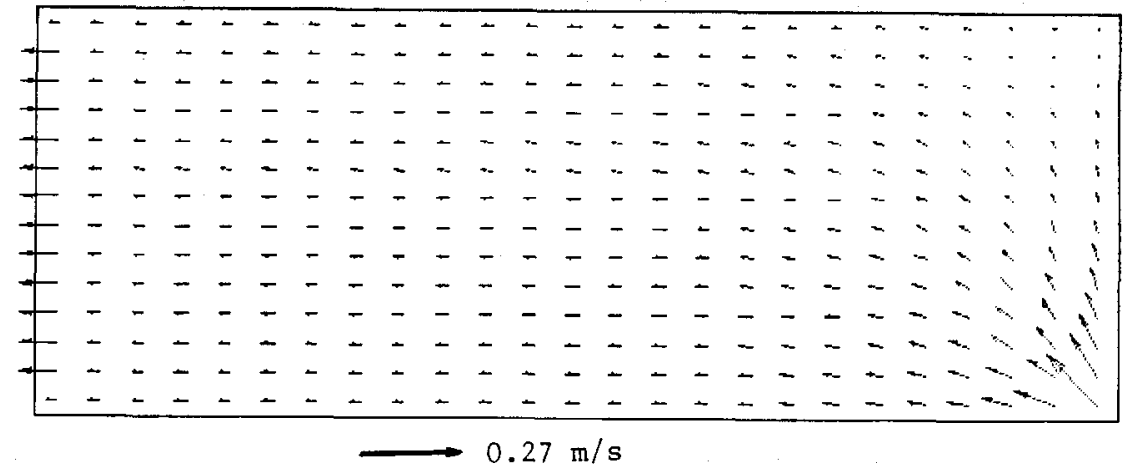

Fig. 6.

Velocity vectors at exit plane $(z=0.75 H)$ for case 2.

Fig. 7.

Velocity vectors near bottom plane $(z=$ $0.1 H)$ for case 2 .

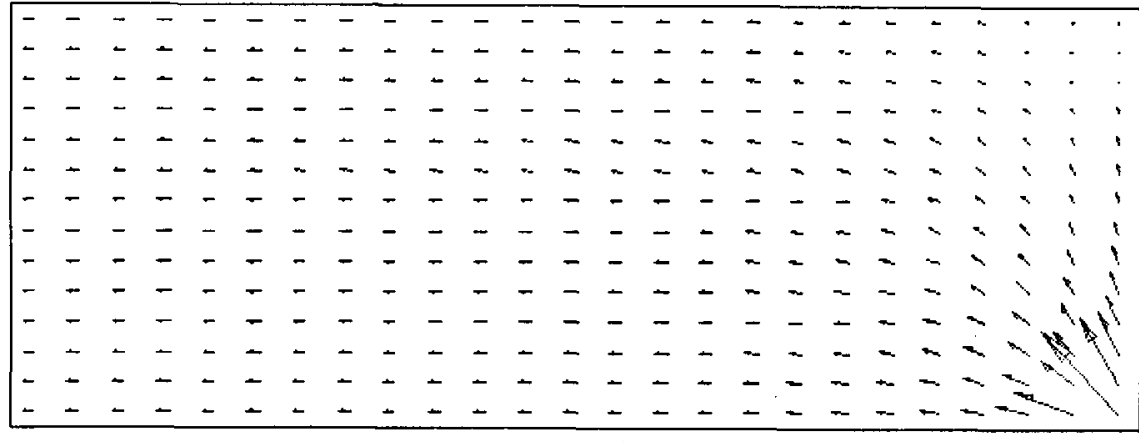

$0.30 \mathrm{~m} / \mathrm{s}$

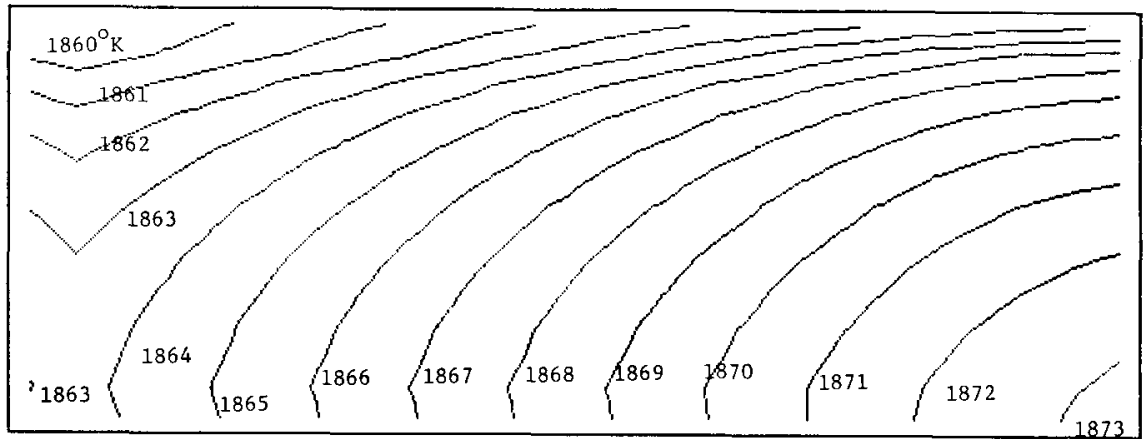

Fig. 8.

Map of temperature at exit plane $(z=$ $0.75 H$ ) for case 2 .

Fig. 9.

Map of temperature near bottom plane $(z=0.1 H)$ for case 2 .

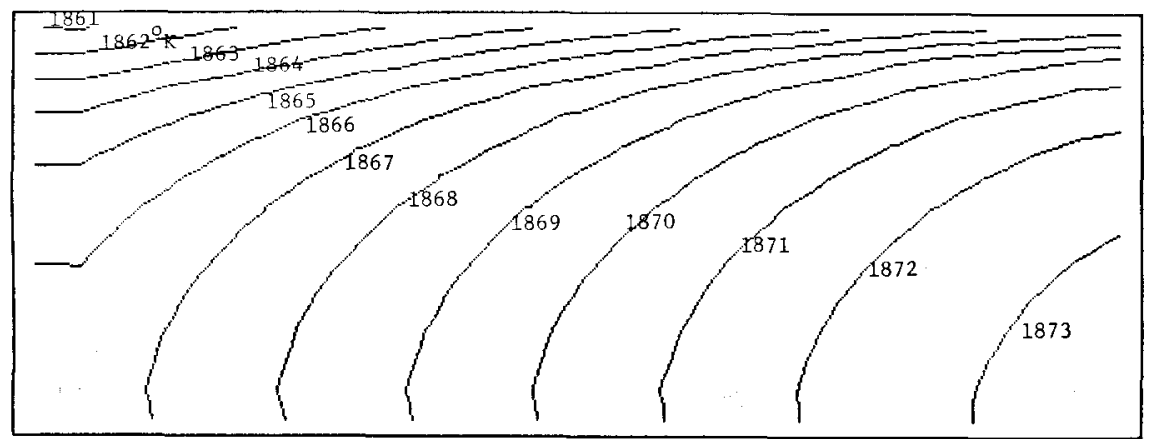

value in the bulk, and partly due to the damping of the mean flow by the constant field. It is also seen that the heat lost in case 5 is much smaller than in case 4 . The reason for this is not immediately obvious. One possible explanation may be that it is due to the progressively dominant influence of the vertical velocity component relative to the mean flow in case 4 as the mean flow is damped by the higher (constant field) away from the inlet region.

The following Figs. 12 to 15 depict the behavior of the inclusion particles. It should be recalled that in contrast to the earlier work by the authors ${ }^{4,5)}$ and others such as Tacke and Ludwig, ${ }^{13)}$ in the present formulation an allowance has been made for the coalesence, flotation and absorption of the inclusion particles at the top slag. This is an important distinction, because the imposition of a magnetic field will tend to reduce turbulence and hence the rate of inclusion coalescence.

In all these calculations, we have assumed a given initial distribution of the inclusion particles, given previously in Table 2. The ordinate appearing in Figs. 12 to 15 represents the relative inclusion density normalized with respect to the initial values i.e. the 


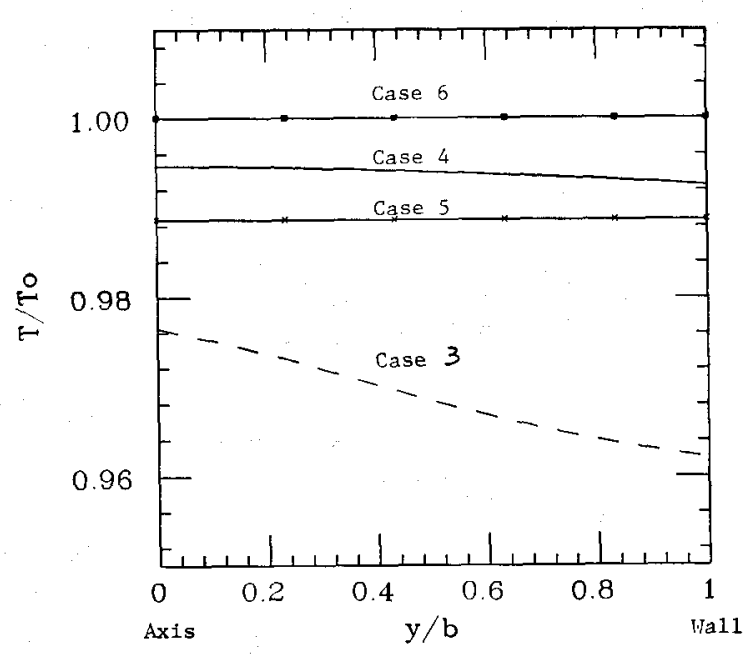

Fig. 10. Temperature variation between axis and wall at exit plane $(z=0.75 H)$.

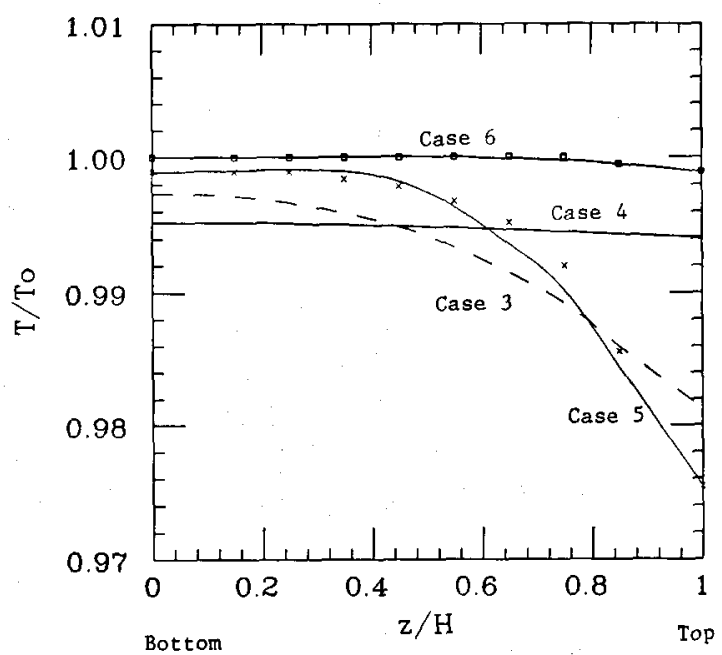

Fig. 11. Vertical variation of temperature at mid plane $(x=0.5 L)$.

residual ratio, while the abscissa is the time normalised by the nominal residence time in the tundish which is defined as:

$$
t^{*}=\frac{\text { Volume of fluid in tundish }}{\text { Volumetric flow rate }}
$$

In these calculations, inclusions will be removed from the system due to flotation and both generated and destroyed by coalescence. In all cases, the magnetic field (where present) are constant and oriented along the transverse direction.

Fig. 12 shows the dimensionless number density of $100 \mu$ inclusion particles close to the inlet, with a constant vertical field as a parameter. It is of interest to note that the imposition of a magnetic field will actually diminish the effectiveness of inclusion removal in this region. This bchavior is reasonable, because coalescence is impeded by the suppresion of turbulence. Furthermore, in the absence of magnetic field the recirculating flow will represent additional mechanism for transporting inclusions to the top surface.

Fig. 13 shows the corresponding plot near the exit.

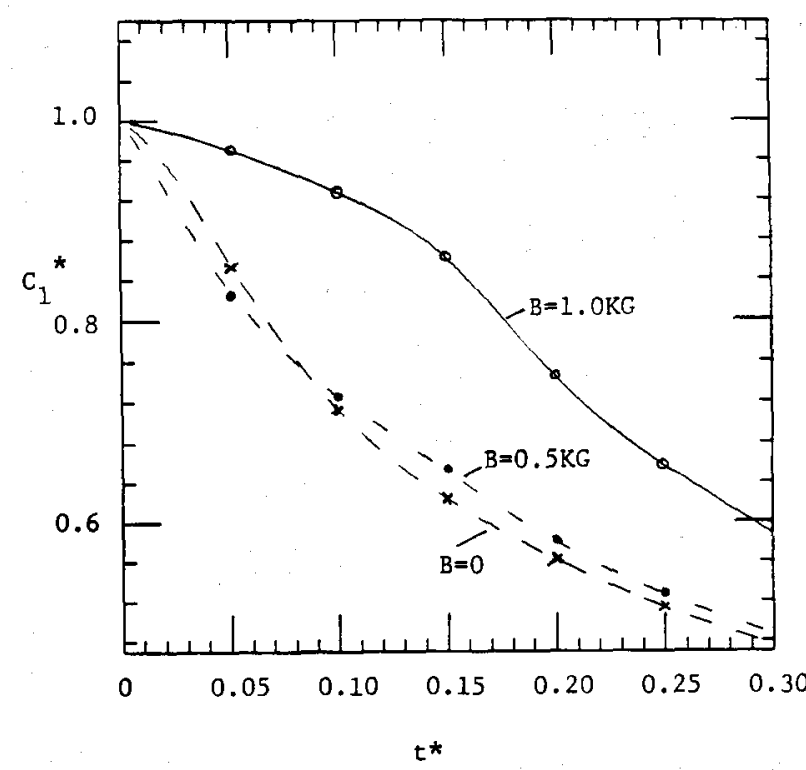

Fig. 12. Variation of dimensionless number density of $100 \mu \mathrm{m}$ inclusion particles with time at a point near inlet $(x=0.1 L, y=0.1 b, z=0.75 H)$.

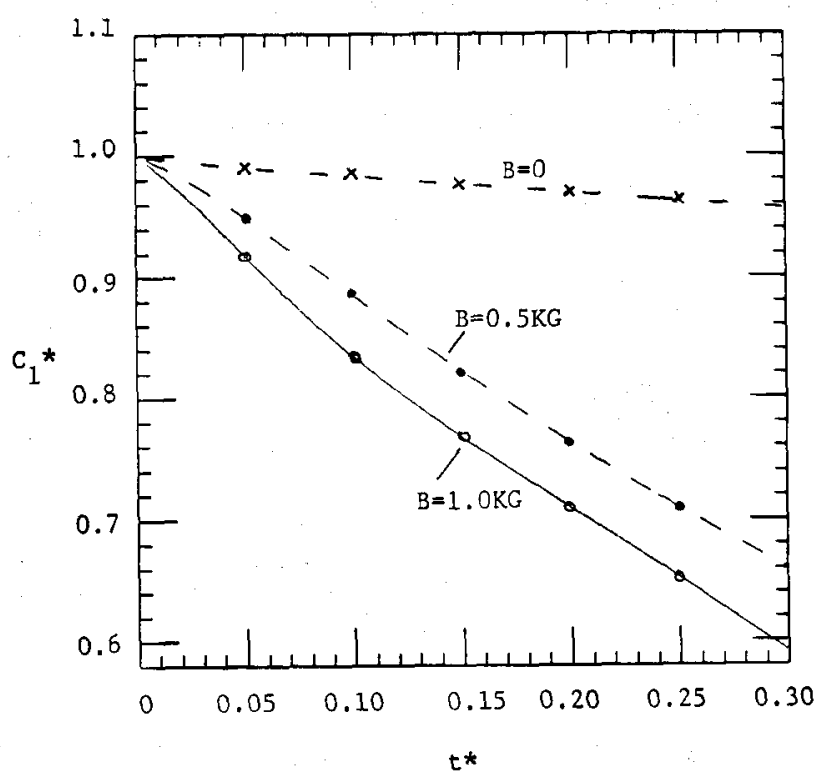

Fig. 13. Variation of dimensionless number density of $100 \mu \mathrm{m}$ inclusion particles with time at a point near exit $(x=0.99 L, y=0.1 b, z=0.75 H)$.

Here the imposition of a magnetic field will significantly improve matters. This result again is consistent with physical reasoning, because an approach to plug flow occasioned by the presence of magnetic field will promote the effective flotation of the inclusion particles.

Figs. 14 and 15 show the corresponding results for the smaller, $20 \mu$ inclusion particles. The behavior of these exhibits qualitatively similar trends; however the extent to which inclusions are being actually removed in these systems remains very low. Indeed, in the majority of cases these tundishes should not be used as inclusion removal devices. 


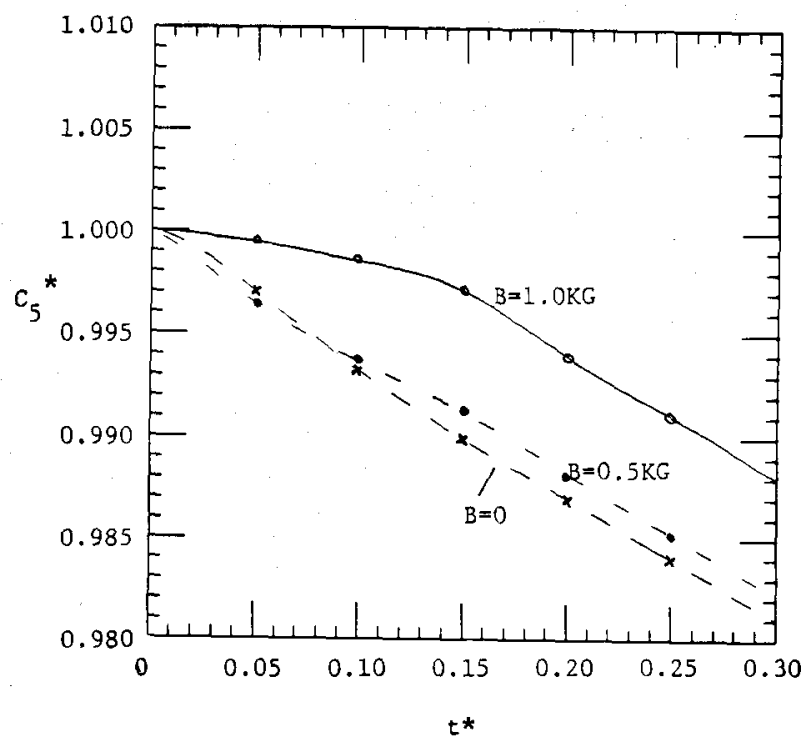

Fig. 14. Variation of dimensionless number density of $20 \mu \mathrm{m}$ inclusion particles with time at a point near inlet $(x=0.1 L, y=0.1 b, z=0.75 H)$.

\section{Discussion}

The principal findings of the work based on the results presented above may be summarized as follows:

(1) In the absence of flow control arrangements, severe flow uniformities may occur in shallow tundishes, which in turn may impose significant variations in the temperature of the metal stream entering the mold of the caster. These problems are likely to become more serious as the mold dimensions are increased, particularly in the production of wide strip or plates.

(2) Electromagnetic flow control through the imposition of horizontal or vertical magnetic fields, can be of great help in minimizing flow maldistribution and horizontal temperature variations in the system. The precise nature of the applied field may play an important role in "fine tuning" the operation.

(3) The effect of magnetic field on inclusion removal is less clear. Traditional tundishes may function quite effectively as "filters" for the removal of larger inclusion particles. In shallow tundishes, the melt residence time tends to be too short for this function to be effectively performed. The imposition of electromagnetic fields will minimize turbulence and hence the coalesence of inclusion particles; at the same time the more uniform residence time of the melt will aid the flotation of the larger particles. In any case, the overall effect is not likely to be significant, which further underlines the need to provide very clean steel to the systems.

The results have shown that appropriately oriented magnetic field would straighten out flow, enhance temperature uniformity and promote inclusion removal. The work presented relied on the application of well established physical laws embodied in the Navier-Stokes' equations and Maxwell's equations so that the conclusions reached should be regarded as

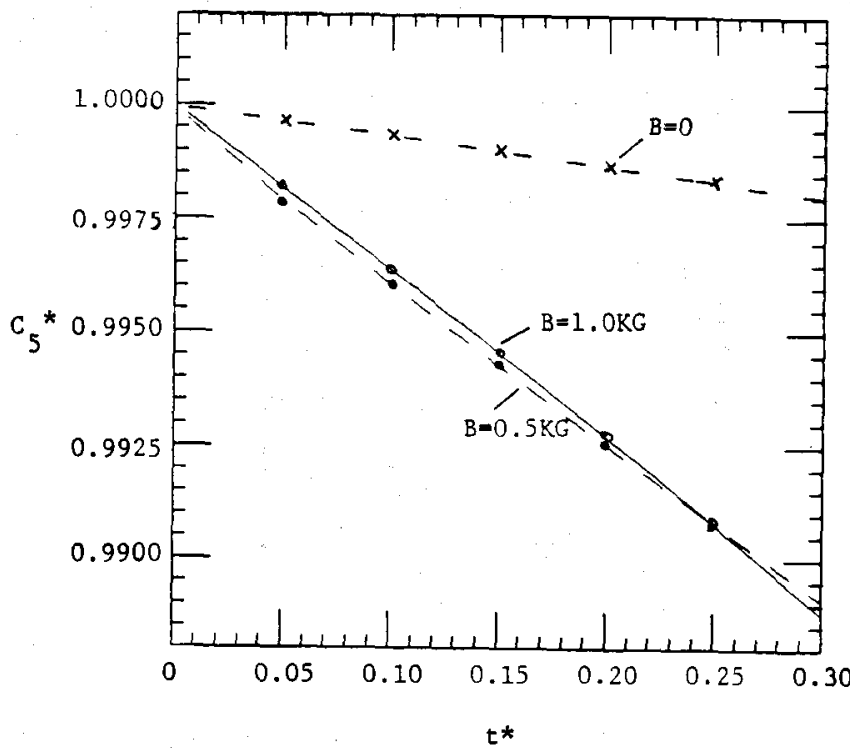

Fig. 15. Variation of dimensionless number density of $20 \mu \mathrm{m}$ inclusion particles with time at a point near exit $(x=0.1 L, y=0.1 b, z=0.75 H)$.

fairly reliable.

While no computed results have been presented in this regard, it is also fair to assume that imposition of appropriately oriented magnetic fields should be useful also for damping out surface waves or disturbances caused by start-up or changes in the flow conditions.

The physical problem of how these fields should be generated or their extent of attenuation and possible non-uniformity (in the presence of massive conductors) still has to be solved. Nevertheless, the generation of fields of the order of 1 Kilogauss should be readily accomplished, even under conventional steel processing conditions.

\section{Conclusions}

A mathematical model has been developed to represent fluid flow, heat transfer, inclusion transport and electromagnetic phenomena in shallow tundishes, appropriate for supplying molten metal to novel thin slab or strip casting systems. This model is an extension of previously published work by the authors in this field specifically in the following respects.

(1) We have examined the effect of magnetic field orientation, including that of spatially variable fields on the melt flow and temperature distribution in the system.

(2) We studied the variation of temperature in the horizontal plane, entering the caster explicitly.

(3) We examined the behavior of inclusion particles, with an allowance for coalescence in the turbulent regions.

\section{Nomenclature}

$b$ : Half width of tundish

$B$ : Magnetic field

$B_{o}$ : Magnitude of magnetic field

$C_{i}$ : Number density of particles of type $i$ characterized by size 
$C_{i s}$ : Number density of particles of type $i$ just below free surface

$C^{*}$ : Inclusion residual ratio (number density/ initial value)

$c_{p}$ : Specific heat

$E$ : Electric field

$f_{i j}$ : Coalescence frequency between particles of types $i$ and $j$

$F_{e}$ : Electromagnetic force per unit volume

$g$ : Gravitational acceleration

$H$ : Depth of tundish

$J:$ Current density

$k$ : Turbulent kinetic energy

$L:$ Length of tundish

$p$ : Static pressure

$q_{i}$ : Flux density of particles of type $i$ at surface

$r_{i}$ : Radius of particles of type $i$

$R_{i j}$ : Sum of radii of coalescing particles of types $i$ and $j$

$t$ : Time

$t^{*}$ : Dimensionless time (time/nominal residence time)

T: Temperature

$T_{0}$ : Inlet temperature

$u, v, w$ : Velocity components along $x, y, z$ directions, respectively

$w_{p i}$ : Terminal rising velocity of particles of type $i$

$x, y, z$ : Coordinates along the length, width and depth of tundish, respectively

$\varepsilon$ : Rate of dissipation of turbulent energy

$\kappa$ : Electrical conductivity

$\mu:$ Laminar viscosity

$\mu_{\text {eff }}:$ Effective viscosity

$\mu_{t}$ : Turbulent viscosity

$\nu:$ Kinematic viscosity

$\rho:$ Melt density

$\rho_{p i}$ : Density of particles of type $i$ $\sigma:$ Prandtl number

$\sigma_{c i}$ : Schmidt number for particles of type $i$

$\phi:$ Electric potential

\section{REFERENCES}

1) J. P. Birat: Proc. Conf. on Restructuring Steel Plants for the Nineties, Inst. Metals, London, (1986), 140.

2) J. D. Naumann and B. Love: "Controlling the Shape of Cast Stainless Steel Strip ", Int. Symp. on Near Net-Shape Casting Strip, 89th Annual Meeting of CIM, Toronto, (1987).

3) J. K. Brimacombe and I. D. Samarasekera: Proc. Int. Symp. on Casting of Near Net Shape Products, Metall. Soc., Warrendale, PA, (1988), 3.

4) O. J. Ilegbusi and J. Szekely: Steel Res., 59 (1988), No. 9, 399.

5) O.J. Ilegbusi and J. Szekely: Proc. Conf, on Casting of Near Net Shape Products, Metall. Soc., Warrendale, PA, (1988), 63.

6) J. Szckely and O.J. Ilegbusi: "Magnetic Streamlining and Flow Control in Tundishes", U.S. Patent No. 87151, (1987).

7) S. L. Soo: Fluid Dynamics of Multiphase System, Ginn Blaisdel, Waltham, MA, (1967).

8) K. Shirabe: "A Mathematical Model of RH Vacuum Degassing System ", M.S. Thesis, MIT, (1981).

9) K. Shirabe and J. Szekely: Trans. Iron Steel Inst. Jpn., 23 (1983), 465.

10) G. Saffman and J. S. Turner: J. Fluid Mech., 15 (1975), 522.

11) B. E. Launder and D. B. Spalding: Mathematical Models of Turbulence, Academic Press, London, (1972).

12) B. E. Launder and D. B. Spalding: Computer Methods in Applied Mech. and Eng., 3 (1974), 269.

13) K. Tacke and J. Ludwig: Steel Res., 58 (1987), No. 6, 262.

14) D. B. Spalding: Mathematics and Computers in Simulation, XIII (1981), 267.

15) S. V. Patankar and D. B. Spalding: Numerical Heat Transfer and Fluid Flow, McGraw-Hill, New York, (1980). 